\title{
Diffusion of Technologies and Social Behavior
}

With 107 Figures and 56 Tables

Springer-Verlag

Berlin Heidelberg NewYork

London Paris Tokyo

Hong Kong Barcelona Budapest 


\title{
Chapter 20
}

\section{Determining the Service Life Cycle of Computers}

\author{
Theodore Modis and Alain Debecker
}

\subsection{Introduction}

Nowadays most people in business are familiar with the application of logistic growth to the sale of products even if they ignore its mathematical formulation. They all use a bell-shaped curve to refer to a product's life cycle. The corresponding S-curve, for a product's cumulative sales, is also something with which they feel comfortable.

Business people are, however, less in tune with the mortality of their products. Hardware maintenance contracts for computers closely follow systems sales. At the beginning the number of contracts increases at the same rate as sales, but instead of the familiar S-curve, contracts reach a peak and then slowly start declining as machines become old and obsolete. In Figure 20.1 we see the familiar forms: the sales life cycle, the corresponding S-curve of cumulative sales, and the less familiar service life cycle. Notice that the last graph peaks only after the product has completed its sales life cycle. Qualitatively the business world is aware of the service life cycle. They refer to it as the product's end-of-life curve. Quantitatively, however, they fall 

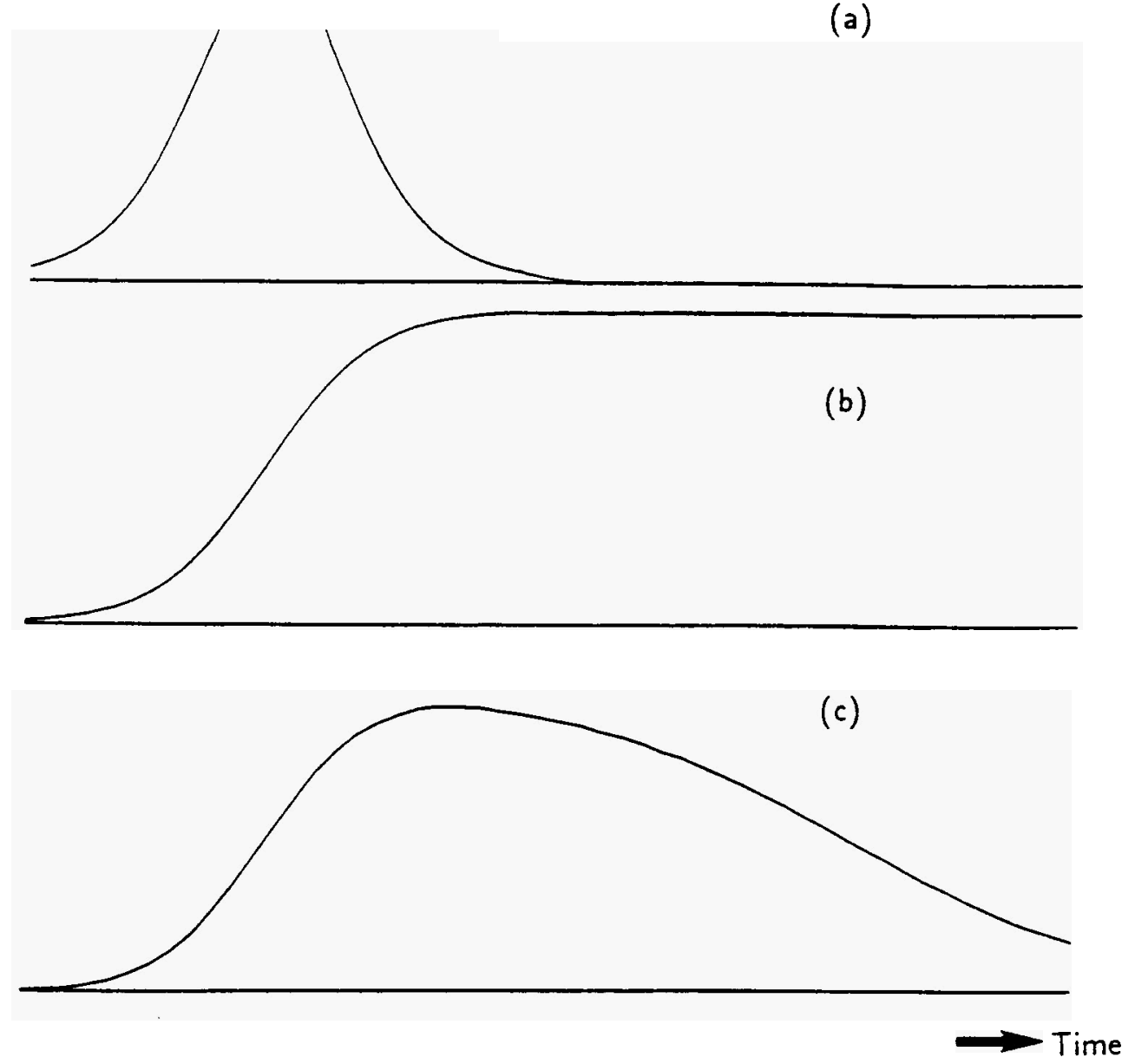

Figure 20.1. (a) Life cycle of sales, (b) Cumulative number of sales, (c) Number of active service contracts at a given time.

short of being able to predict when the product will peak and at what rate revenue will decline afterwards.

The life cycles of products are becoming shorter. A specific computer model used to sell for 4-5 years in the 1970s; they now only sell for 1-2 years. Service life cycles have been known to last for 12-14 years. By how much are they going to decrease in the future? 
A recent article in the Harvard Business Review addresses this question (Potts, 1988). Graphs and figures liven un the discussion, but the treat.ment remains qualitative and rather pictorial. It corroborates the intuition but does not help with quantitative forecasts. Determining the service life cycle is interesting for other reasons besides the ever-increasing importance of service revenue. It provides knowledge of the installed base, its actual and future size, which is crucial in marketing strategies for add-ons (follow-up sales in terms of accessories, upgrades, etc.), software, and a variety of services. This is why we propose a quantitative approach for determining a product's life span as opposed to its sales life cycle only.

\subsection{Logistic Diffusion}

Logistic growth formulated in the Volterra-Lotka equations accurately describes situations where a niche is being filled under natural competition, be it an ecological or a market niche. The situation becomes more complicated when one is interested in tracking down the survivors of a generation over time. The total number of units sold of a particular product increases with time reaching its ceiling at the end of the product's life cycle. The number of products in use, however, never reaches the same ceiling as there is a certain mortality among the products sold. Out of a hundred units sold in month one, 98 may be still "alive" a year later but only 50 in five years time. This erosion comes from aging and obsolescence, and sets in right from the beginning. While populations grow happily along their S-shaped birth curve, they are at any point in time subject to a certain mortality.

Mathematically the case calls for a convolution function. The folding of logistic growth with a mortality function. What shape should the latter have? Sales follow a logistic growth, namely the rate of sales over time, $P(t)$, proceeds along a bell-shaped curve of the form:

$$
P(t)=\frac{M}{\left(1+e^{-\alpha\left(t-t_{0}\right)}\right)\left(1+e^{\alpha\left(t-t_{0}\right)}\right)}
$$

where $M, \alpha$ and $t_{0}$ are constants. To introduce mortality we begin with the simplest possible assumption of a constant percentage decay rate, in other words an exponential decay:

$$
\frac{1}{R(u)} \frac{d R(u)}{d u}=-k
$$




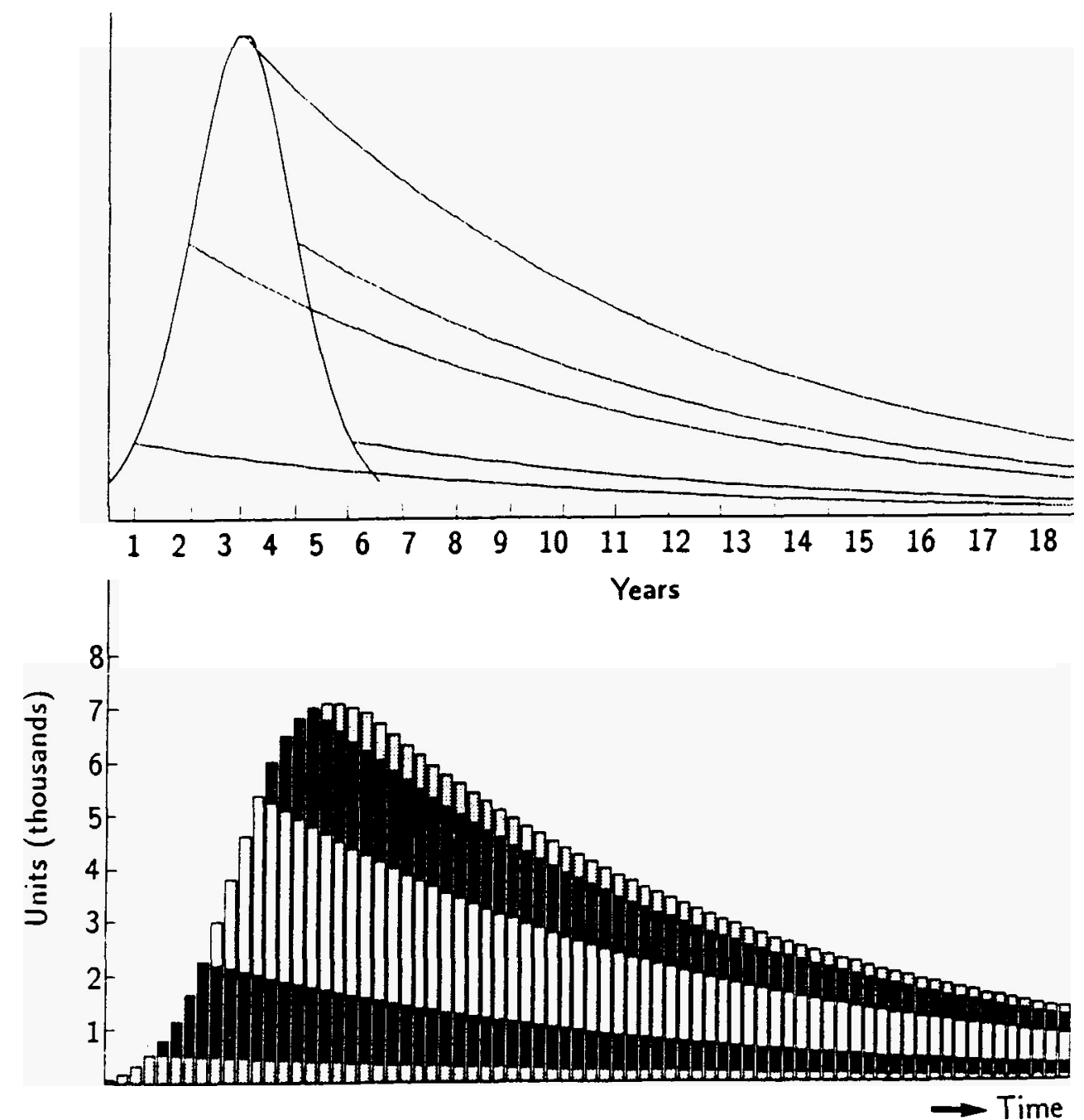

Figure 20.2. Above: life cycle outlining sales of service contracts and exponential mortality rates over a five-year period. Below: the integrated number of contracts with finer time bins (trimesters). The overall envelope represents total survivors at a given time.

where $R(u)$ is the remaining contracts of age $u$, and $k$ is a constant. The convolution function is given below and graphically it is shown in Figure 20.2. 


$$
\begin{aligned}
Q(t) & =\int_{0}^{\infty} P(t-u) R(u) d u \\
& =\int_{0}^{\infty} \frac{M e^{-k u}}{\left(1+e^{\alpha\left(t-u-t_{0}\right)}\right)\left(1+e^{-\alpha\left(t-u-t_{0}\right)}\right)} d u
\end{aligned}
$$

When we first applied this formulation to real data we obtained good results, particularly for old products for which the sales life cycle had finished years ago and service contracts were already beyond their peak and declining. However, two difficulties soon emerged. One was related to relatively young products where the rate of decay was so low that it implied they would be around for a very long time. This fact contradicts the increasingly rapid cycling of products witnessed in the computer industry. The second difficulty was more revealing. When tracking decay rates in a number of areas we found that they were not constant over time. These observations prompted us to raise the level of sophistication for mortality, i.e., to try a second degree function for the decay rate:

$$
\frac{1}{R(u)} \frac{d R(u)}{d u}=a R(u)+b
$$

where $a$ and $b$ are constants. Setting $N=-b / a$ we can rewrite this expression as

$$
\frac{d R}{d u}=-a R(N-R)
$$

which unveils the logistic nature of the assumed form. Furthermore, we can eliminate one constant by setting $N=1.0$ since the mortality ceiling is $100 \%$.

We have now arrived at a logistic mortality, however, it was not entirely circumstantial. An older study on the appearance and survival of supertankers had revealed to us an exemplary S-shaped mortality for that "species". In addition, Marchetti has investigated human mortality (Marchetti, 1990) and has also established logistic laws for the process. However, what is important is that the logistic mortality better fits the data on computer service contracts we were trying to describe. The new convolution function described below involves five parameters, three from the logistic growth of contract "births", equation (20.1), and two from the logistic mortality, equation (20.3), ( $u_{o}$ is an integration constant). This is graphically depicted in Figure 20.3. 

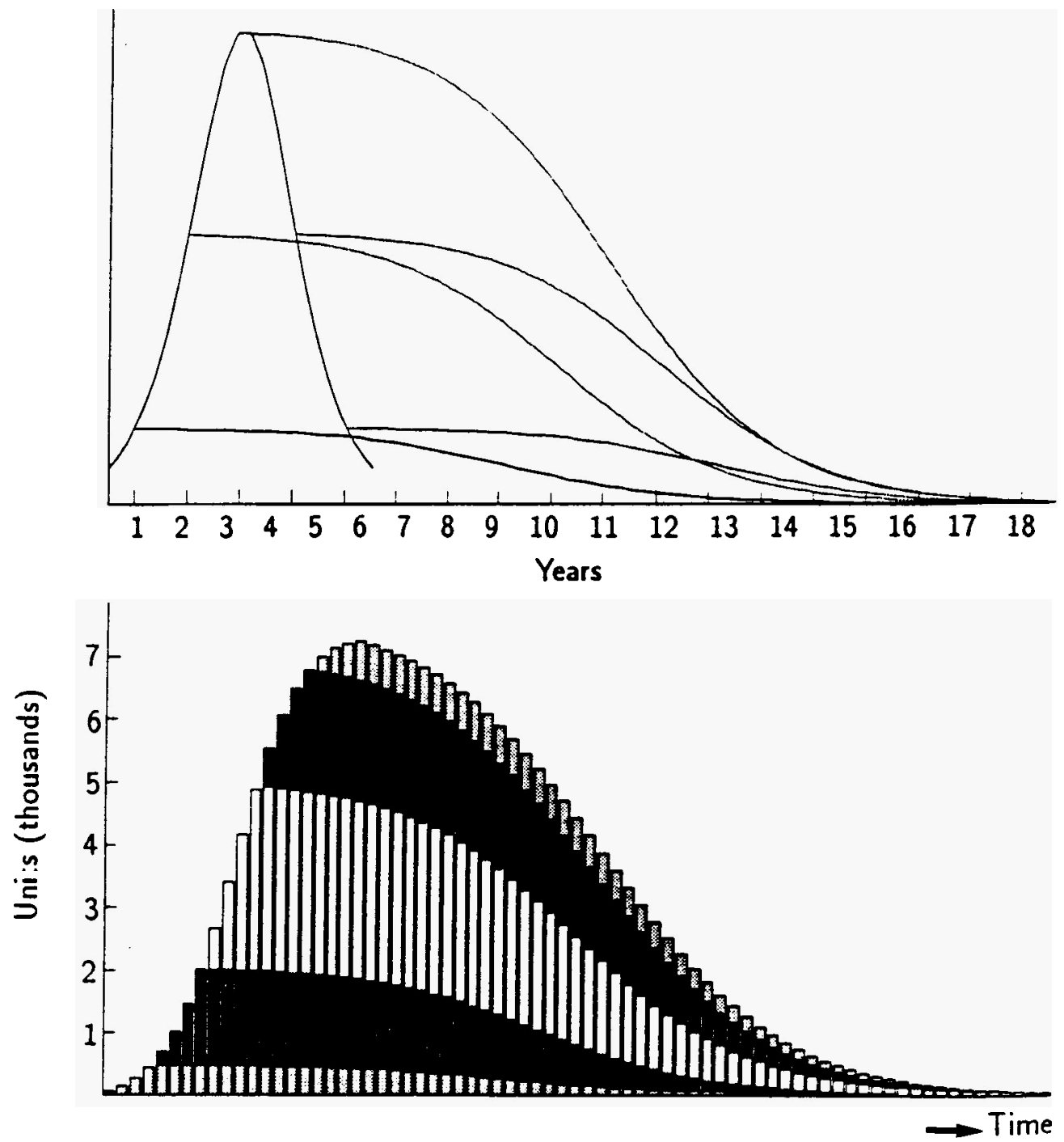

Figure 20.3. Above: life cycle outlining sales of service contracts and logistic mortality rates over a five-year period. Below: the integrated number of contracts with finer time bins (trimesters). The overall envelope represents total survivors at a given time. 


$$
\begin{aligned}
Q(t) & =\int_{0}^{\infty} P(t-u) R(u) d u \\
& =\int_{0}^{\infty} \frac{M}{\left(1+e^{\alpha\left(t-u-t_{0}\right)}\right)\left(1+e^{-\alpha\left(t-u-t_{0}\right)}\right)\left(1+e^{a\left(u-u_{0}\right)}\right)}
\end{aligned}
$$

\subsection{Applications}

Using equation (20.4) in forecasting or business planning implies that the five parameters have to be determined. To do this experimentally one needs at least five measurements - monthly or quarterly data points on active contracts. In order to limit the uncertainties involved in logistic fits one must have many data points. Consequently parameter determination becomes a fitting procedure where the five unknowns are to be determined from many more than five data points. The procedure we used is one of a $X^{2}$ minimization. The $X^{2}$ is formed as follows:

$$
X^{2}=\sum_{i} \frac{\left(D_{i}-Q_{i}\right)^{2}}{w_{i}}
$$

where $D$ is the data array, $Q$ from equation (20.4), $w$ a series of weights to be supplied, and $i$ the index of time bins.

A function minimization program was used to search for the values of the five parameters. The weights were usually taken to be uniform, but in some cases they were adjusted through business knowledge. An example is shown in Figure 20.4. For this older computer model, even though we are missing the early data, we have enough points (monthly data for six years) to make a reliable determination. In fact, by ignoring the most recent year, the last 12 points from the fit have a negligible effect on the parameter values. Repeating the operation, i.e., dropping the last 24 points, still replicates closely the parameter values originally found. In conclusion, we can say that as long as the historical data go far enough to hint at the decline beyond the peak, the parameter determination shows a remarkable stability.

We did, however, encounter two unexpected difficulties.

\subsubsection{Young products}

The first difficulty involved young products, i.e., products for which the historical data have not yet reached the peak. In these cases a 4- or 5parameter fit, involving equations (20.2) or (20.4) respectively, produced $a$ 


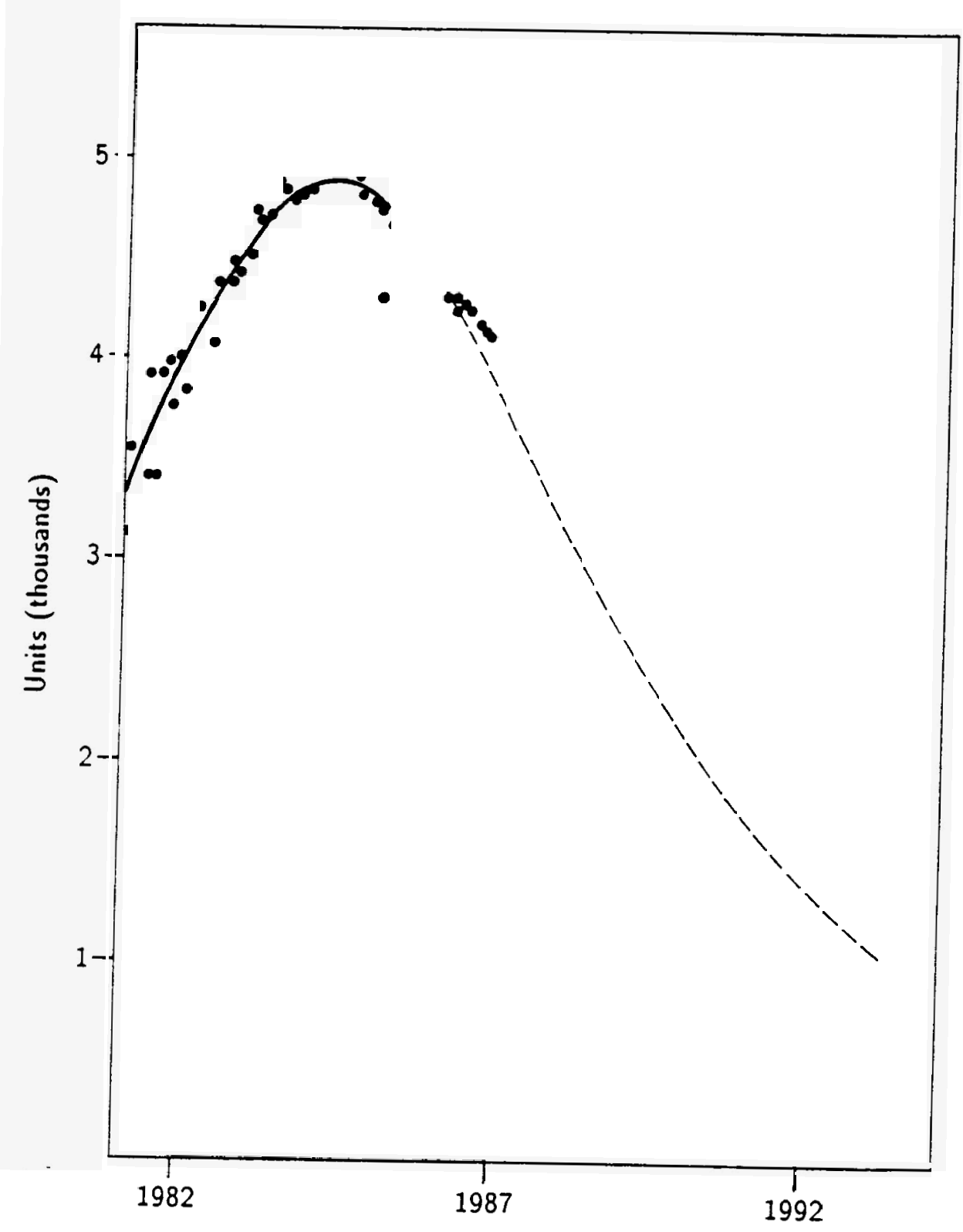

Figure 20.4. Monthly data and fit for the hardware service contracts of an old computer model. Each point represents the number of active contracts at the time. The fit (solid line) is based on the data up to the end of 1985, two years before the end of the historical window. The dotted line is the extrapolation of the convolution function determined from the fit. 


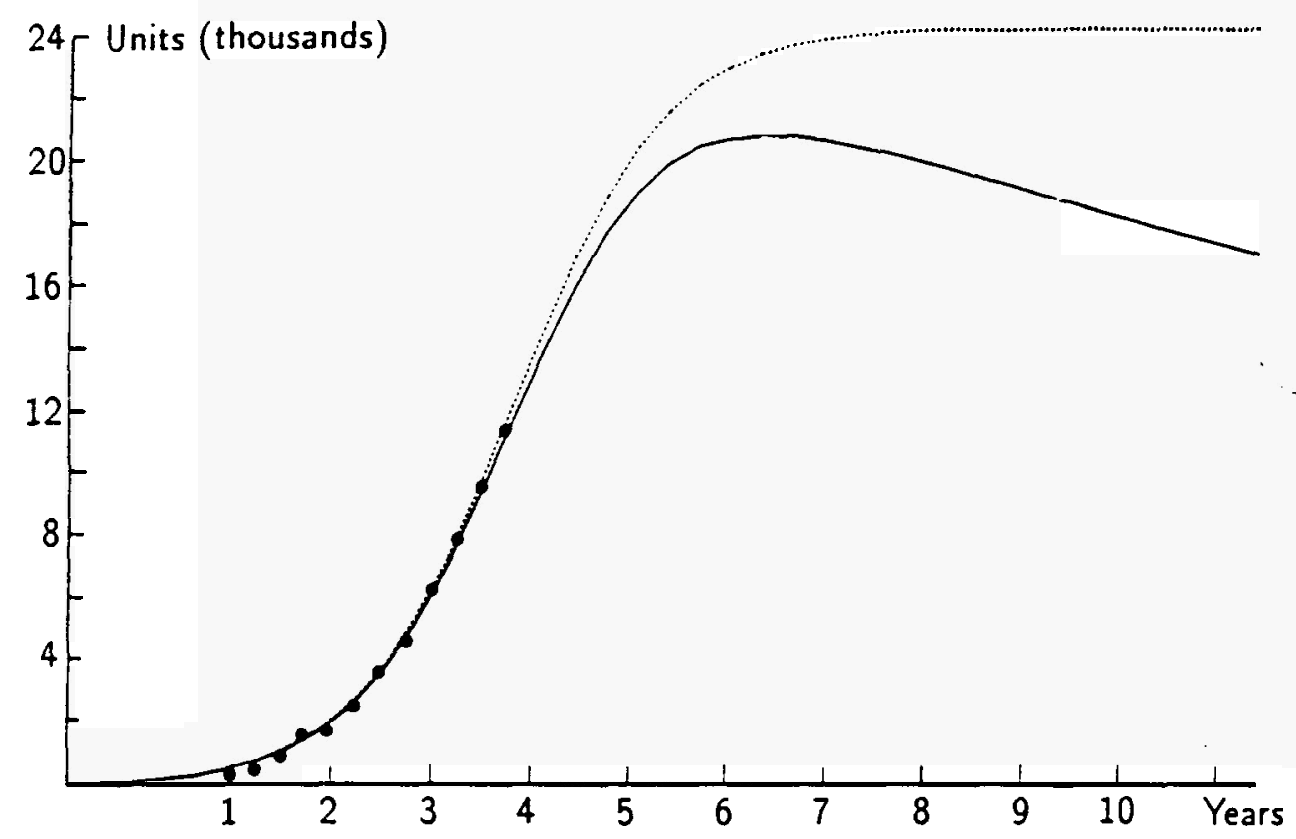

Figure 20.5. Trimesterly data and fit for the active contracts of a recent computer model. The fitting program yielded a null mortality (dotted line). The solid line suggests the realistic evolution taking mortality into account.

null mortality most of the time. The best fit found by the program would be a simple logistic growth with zero mortality. This was seen in terms of the fact that the difference between contracts sold and contracts active was very small in the early days of a new product. The program could not determine mortality parameters if a simple 3-parameter growth logistic could describe the data just as well (see Figure 20.5). Although this is a comforting feedback on the robustness and stability of the parameter determination method, it precluded the determination of contract mortality for young products.

At the same time it inspires a resolution to the problem through factorization. We adopted a two-step procedure. First we fit the contract sales data, i.e., the appearance of new contracts, to a simple 3-parameter logistic. Once we determine the ceiling this way, we fit the active-contract data to the convolution function (20.2) or (20.4), but this time with the maximum $M$ fixed at the value already determined from the sales fit. Clearly the procedure demands knowledge of the "birth" of contracts as well as the contracts active at a given time. 


\subsubsection{Speeding up the computation}

The second difficulty concerned the computer time required. Working for a computer company one is often spoilt because there are usually unlimited computing resources. Nevertheless, human nature is such that having does not quench greed. When we realized that the minimization of a $X^{2}$ involving the convolution of two logistic functions, with five parameters needing to be determined, required more than a few minutes of real time computing, we found the situation unacceptable. Rather than searching for programming tricks to reduce the computing time we adopted a mathematical trick. The calculation of the exponentials involved in the logistic expression takes a long time to calculate. Logistic growth, however, can also be obtained through a simple hyperbola and the recursive relation (Meade, 1985):

$$
x_{n+1}=\frac{x_{n}}{a x_{n}+b}
$$

where $a$ and $b$ are constants and the third constant is the starting value $x_{0}$.

The new approach then is to construct an array, element by element, using relation (20.6) and a similar one for mortality with two parameters only (the starting value is taken close to $100 \%$, representing mortality at age zero). The five parameters are then determined by using the minimization program to best match the constructed array to the data, the active contract array is shown at the bottom of Figure 20.6. Whenever the data are available in this way, in the full diagonal matrix form, a further factorization becomes possible and mortality can be determined independently from each line (across), while sales keep growing along their life cycle (down).

\subsubsection{Phasing out as a generation}

It is common knowledge that the usefulness of a generation of computer models decreases with time not because of aging, e.g., material fatigue resulting in frequent breakdowns, but due to obsolescence. Computer models do not drop out of use individually like used cars. They phase out together as their technological generation becomes outdated.

This phenomenon is confirmed here in a formal way. From Figure 20.3 we see that the number of models in operation declines logistically from the day of sales. The end-of-life point, taken as the $1 \%$ of sales that remains at a certain date, is reached at approximately the same date for all models. People who bought the very first models will keep them for up to 16 years. Those who bought the last few models sold say six year later, will only 


\section{Contract}

"births"

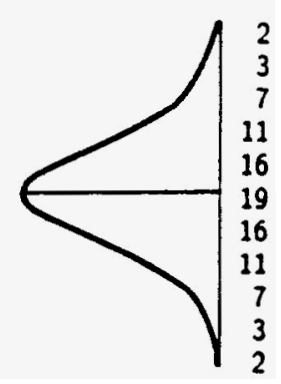

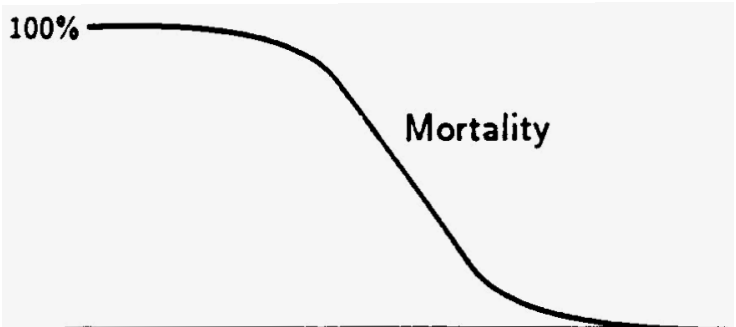

$\begin{array}{llllllllllllll}2 & 2 & 1 & 1 & 1 & 1 & 1 & 1 & 1 & 1 & 1 & 1 & 0 & 0\end{array}$

$$
\begin{array}{rrrrrrrrrrrrr}
3 & 3 & 3 & 3 & 2 & 2 & 2 & 2 & 1 & 1 & 1 & 1 & 1 \\
& 7 & 6 & 5 & 5 & 4 & 4 & 3 & 3 & 3 & 3 & 2 & 2 \\
& 11 & 10 & 9 & 8 & 7 & 7 & 6 & 5 & 5 & 4 & 4 \\
& & 16 & 15 & 13 & 12 & 11 & 10 & 9 & 8 & 7 & 6 \\
& & & 19 & 17 & 15 & 14 & 12 & 11 & 10 & 9 & 8 \\
& & & & 16 & 15 & 13 & 12 & 11 & 10 & 9 & 8 \\
& & & & & 11 & 10 & 9 & 8 & 7 & 7 & 6 \\
& & & & & & 7 & 6 & 5 & 5 & 4 & 4 \\
& & & & & & & 3 & 3 & 3 & 3 & 2 \\
& & & & & & & & 2 & 2 & 1 & 1
\end{array}
$$

$\begin{array}{llllllllllllll}2 & 5 & 11 & 21 & 35 & 51 & 61 & 67 & 68 & 63 & 59 & 55 & 47 & 42\end{array}$

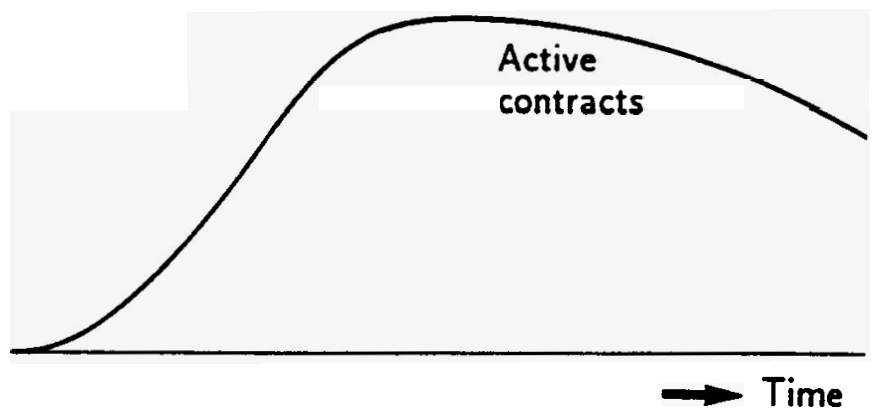

Figure 20.6. The full matrix of contracts for a hypothetical model. The diagonal elements represent initial contract sales. Projected vertically on the left they give rise to the life cycle. Each horizontal array displays mortality over time. The bottom line represents active contracts as a function of time.

keep them ten years. This generation of computers had a sales life-cycle of six years and a service life-cycle of 16 . Perhaps unfairly, the models fabricated last, even though perfected and more reliable are endowed with shorter lifetimes. 


\subsection{Conclusion}

Unlike cumulative product sales, service contracts display a mortality over time. Computer service contracts are best described and forecast through the use of a convolution function of two logistics: one representing the growth of contract sales (three parameters) and the other representing contract mortality (two parameters).

For young products it is recommended to factorize the process into two fits, births of contracts first and active contracts afterwards. To keep computing time within convenient limits, one should use a recursive hyperbolic relationship instead of the analytic function involving exponentials.

Computer models in operation phase out independently of the date they were sold. As their technological generation becomes obsolete, early sales and late sales all drop out of use at the same time.

Beyond hardware service revenue forecasts, the approach offered here helps determine the strategically important installed base which is the market for add-ons, software, and a multitude of other services.

\section{References}

Meade, 1985, Forecasting Using Growth Curves - An Adaptive Approach, Journal of the Operational Research Society 36(12):1103-1115.

Marchetti, C., 1990, Longevity - An operational definition, private communication.

Potts, G.W., 1988, Exploit Your Product's Service Life Cycle, Harvard Business Review 66(5):32-36 (September-October). 